\title{
Decision experience in hyperchoice: the role of numeracy and age differences
}

\author{
Natasha Peterson ${ }^{1} \cdot$ Jiuqing Cheng $^{1}$ \\ Published online: 14 September 2020 \\ (C) Springer Science+Business Media, LLC, part of Springer Nature 2020
}

\begin{abstract}
Due to new technologies, a profusion of products is released onto store shelves and the Internet, resulting in a special choice condition termed hyperchoice. Past research on whether hyperchoice deteriorates decision experience is mixed. The present study hypothesizes the experience in the scenario of hyperchoice may be moderated by individual characteristics, including numeracy and age differences. A total of 116 older adults and 112 younger adults were recruited from Amazon Mechanical Turk. Along with the Rasch-based numeracy scale, each participant completed a consumer and a gamble choice task. In both tasks, the number of options being presented to participants was manipulated to create a hyperchoice condition (sixteen options) and a simplechoice condition (four options). Dependent variables were post-choice difficulty and satisfaction. Multiple regressions were performed with SPSS 24.0 to test the hypothesis. As a result, hyperchoice was related to greater decision difficulty in both choice tasks. Moreover, there was an interaction between numeracy and hyperchoice in the gamble task. Specifically, whereas higher numerate participants' experienced difficulty and satisfaction were relatively stable between the two choice conditions, lower numerate participants experienced more difficulty and dissatisfaction in the hyperchoice condition than in the simple-choice condition. Additionally, compared to younger adults, older adults reported greater decision difficulty and lower decision satisfaction, regardless of choice condition. The study supported the notion that the specific effect of hyperchoice was moderated by individual factors. The study implied merchants should adopt strategies to ease decision experience and advocated for numeracy education.
\end{abstract}

Keywords Age $\cdot$ Numeracy $\cdot$ Hyperchoice $\cdot$ Consumer choice $\cdot$ Gamble choice $\cdot$ Choice overload

Due to new technologies and competitive pressures, a profusion of new products is released onto store shelves and onto the Internet every day, making consumers of all ages face a glut of purchase alternatives. Accordingly, researchers coined the terminology "hyperchoice" as a condition, similar to "information overload,' in regard to consumerism whereby an 'ever-increasing amount of buying occurs amidst an everincreasing amount of new products, brands, and brand extensions' (Mick et al. 2004). In academic literature, such a phenomenon is also termed choice overload (Scheibehenne et al. 2010), overchoice effect (Gourville and Soman 2005), and too-much-choice effect (Scheibehenne et al. 2009).

Conceptually, hyperchoice may adversely affect decision experience because its excessive number of options

Jiuqing Cheng

jiuqing.cheng@uni.edu

1 Department of Psychology, University of Northern Iowa, Cedar Falls, IA 50614, USA overwhelms decision makers' cognitive capacity and drain people's psychological energy (Iyengar and Lepper 2000; Mick et al. 2004). However, as reviewed below, past research on the effect of hyperchoice on decision experience was not consistent. How hyperchoice affects decision maker's feeling remains unclear. In the global pandemic of COVID-19, as people are largely confined to their homes, individuals are more likely to pursue online shopping and thus face more hyperchoice scenarios. For instance, an Amazon search of 'face masks' results in over 100,000 results-each varying in price, style, count per pack, brand, purpose, reusability and adjustability. Hence, it is more imperative to unpack the effect of hyperchoice. The present study attempts to fill the gap by hypothesizing and testing the interaction between individual characteristics and hyperchoice. Specifically, we aim to show how people's experience in the scenario of hyperchoice may depend on their age and numeracy, two important characteristics that affect intellectual activities and decision making. We present our rationale and relevant literature below. 


\section{Mixed Results of Hyperchoice}

Even though hyperchoice provides abundant alternatives, some studies have found that the scenario also leads to challenges and worse decision experience. In a classical study regarding hyperchoice, Iyengar and Lepper (2000) compared choices of jams and chocolates, where options were as many as 30 and as few as six. Participants felt making choices in the hyperchoice condition more difficult, and subsequently reported lower satisfaction as well as more regret over their choices. The authors of this study believe that the root of these results stemmed from participants having too much freedom of choice, creating more unwanted responsibility (Iyengar and Lepper 2000). Similarly, Larceneux et al. (2007) carried out two experiments applied to the jewelry-watchmaking market and manipulated the number of products. As a result, when faced with more products, participants experienced more negative effects on attraction and desire to make a choice. In addition to choosing tangible consumer goods, recent studies on dating partner (D'Angelo and Toma 2017), vacation destination (Thai and Yuksel 2017) and electricity service provider (Jilke et al. 2016) selections also found greater dissatisfaction and regret when decisions were made in a hyperchoice relative to a simple-choice condition.

Contrary to the findings above, a meta-analysis with 50 published and unpublished experiments revealed that there was no significant impact of hyperchoice (or choice overload) on participants' satisfaction with their choices (Scheibehenne et al. 2010). However, this study also found significant variability in effect size across the experiments included in the meta-analysis, indicating a potential moderating effect in the function of hyperchoice. Chernev, Bocknholt, \& Goodman (2015) performed another meta-analysis based on 51 studies from 21 published articles and found the overall effect of hyperchoice was significant. Most importantly, the study identified two extrinsic factors (choice set complexity and decision task difficulty) and two intrinsic factors (preference uncertainty and decision goal) that moderated the effect of hyperchoice on decision experience. Thus, although the two meta-analysis studies did not agree on the main effect of hyperchoice, both implied the importance of possible moderation effects. In line with this notion, Lee (2017) asked participants to select a backpack out of a variety of options. It was found that although increasing the assortment complexity decreased choice satisfaction in general, such effect was less evident in participants with a higher level of need for variety than in participants with a lower level of need for variety. In other words, the need for variety moderated the effect of hyperchoice on choice satisfaction. The present study aims to continue unpacking the moderation effect on hyperchoice. Specifically, the study focuses on two individual characteristics: age differences and numeracy. As discussed below, we chose these two intrinsic (subjective) factors because they are fundamental and critical to decision making.

\section{Hyperchoice and Age Differences}

Previous research has demonstrated the effect of age differences on decision making, as shown in the comparisons between older adults and younger adults (e.g., Chen and Sun 2011; Dror et al. 1998; You et al. 2019). For example, Chen and Sun (2011) found that compared to younger adults, older adults were more likely to adopt simple heuristic strategy in a simulated yard sale task. With the world's older population growing rapidly (He et al. 2016), fostering a better understanding of how aging affects decision making is increasingly more pertinent to older people themselves, as well as to marketers and to public policy makers. Adult abilities can stay stable for many years; unfortunately, the general pattern for the aging process is decline in memory, processing speed, language, visuospatial skills, and executive functioning abilities (Gluck et al. 2016; Harada et al. 2013). The decline of cognitive abilities may lead to decision difficulty. For example, in a food choice study, older adults had more difficulty in selecting and utilizing relevant nutritional information from a complex environment that contained both relevant and irrelevant information compared to their younger counterparts (Cole and Gaeth 1990). Thus, understanding decision experience in older adults is particularly meaningful given the aging demographic trends.

Hyperchoice applies to medical decision making in the era of aging and can lead to worse decision experience. Take Medicare Part D (drug plans) in the United States as an example. Medicare Part D provides the opportunity for beneficiaries (mostly are over 65) to purchase insurance coverage to pay for the cost of prescription drugs. While such drug plans help the beneficiaries control the cost of buying drugs, one significant issue is the relatively large number of available drug plans to choose from. For example, in 2008, most states provided over 53 plans (Tanius et al. 2009). In 2019, on average, beneficiaries had 48 different plans to select from (Kaiser Family Foundation, 2019). Contrary to the traditional idea that more is better in decision making (Payne et al. 1993), the large number of options was considered to be too complicated by 73\% of older responders (Kaiser Family Foundation 2006). Not surprisingly, the complex choice set of Medicare Part D received criticisms. For instance, as cited in Reed et al. (2008), one older adult proclaimed, "I'm 85, do I have to go through this nonsense? I'm trying to absorb all the information, but it's ridiculous. Not just ridiculous, it's scary. If there was a single card and it was administered by Medicare, and it got the cost of drugs down - wonderful, marvelous".

As previously stated, aging generally leads to systematic declines in cognitive processing. Working memory, a key component to decision making and other intelligent activities, is also sensitive to cognitive decline, which can be problematic when older decision makers need to compare a number of alternatives (Carpenter and Yoon 2012). Thus, in line with the findings in the Medicare example described above, the study predicts that older adults in comparison to younger adults will experience greater difficulty when facing a surfeit of options. 


\section{Hyperchoice and Numeracy}

Making decisions usually involves dealing with numerical information. For example, in consumer choices, people often need to process numerical information such as prices, discounts, and customer ratings. In financial decision making, people need to comprehend probability to evaluate risk. Therefore, the ability to process numerical information is related to decision making. According to Peters and Bjalkebring (2015), numeracy refers to the cognitive ability to understand, process, and utilize numerical information. Moreover, lower numeracy has been found to be associated with biased decisions (Peters and Bjalkebring 2015). For example, in intertemporal choices, lower numerate people were more likely to select the sooner, smaller gains over the later, larger gains, and hence, made more impulsive choices (Sinayev and Peters 2015; Cheng 2020). In gamble choices, lower enumerate participants were more likely to deviate from the expected utility axiom (Jasper et al. 2017). In medication-related decisions, lower numerate people were less likely to adopt the best possible solution because they could not appropriately utilize and comprehend numerical information such as survival rate and treatment effectiveness (Reyna et al. 2009).

Conceptually, with more options, lower numerate people may experience a more difficult time to comprehend and utilize numerical information because doing so may cause them to encounter cognitive overload. Hence, hyperchoice may impose some extra challenges on lower numerate people. As numeracy is increasingly popular in research on decision making (Cokely et al. 2018), the present study aims to advance knowledge of both hyperchoice and numeracy by testing a possible connection between the two.

\section{Hypothesis and Methodological Setting}

This study aims to test the possible moderation effect of age differences and numeracy in people's decision experience in the scenario of hyperchoice. Specifically, as introduced above, the study hypothesizes the negative impact of hyperchoice on decision experience tends to be stronger in older adults than in younger adults, and/or in lower numerate people than in higher numerate people.

In addition to the hypothesis above, the present study aims to advance the knowledge of hyperchoice with two methodological settings (contexts). First, most past studies adopted consumer choice tasks (as an example, see Table 1 in the two metaanalysis studies stated above). The present study examines whether the effect of hyperchoice can be generalized to another decisionmaking domain by adding a gamble choice task.

Second, in past research, a common approach used to test the hyperchoice effect asked participants to make selections/ evaluations between a simple-choice condition (fewer options) and a hyperchoice condition (more options). A possible limitation of such an approach is that participants' feelings might be confounded with factors other than the amount of information. To illustrate, a retired person wants to select a state to relocate. In the hyperchoice condition, the options are twelve Midwestern states. In the simple-choice condition, the options are Florida and Texas. The person may prefer the simple-choice condition because there are not too many options. Alternatively, the person may simply want to find a warm place to relocate, and hence, feels the simple-choice condition is more appealing. In the second scenario, the person's feelings are confounded by climate. To address this issue, the characteristics (besides quantity) should be held at a comparable level between different choice conditions. For instance, in one of the experiments in Iyengar and Lepper (2000), students were asked to select an essay topic from a hyperchoice condition (thirty topics) and a simple-choice condition (six topics). The authors stated, "Careful attention was given to selecting essay topics that were comparable in difficulty." However, it is not clear whether all published studies did so. The control of characteristics or quality was not stated in all previous studies. In the present study, to hold the characteristics of the conditions constant, we present exactly the same options between the simple-choice and hyperchoice conditions. The difference is that in the simple-choice condition, we present four options in each trial and participants go through four trials separately; whereas in the hyperchoice condition, we present sixteen options altogether. Our purpose is to increase the internal validity of our manipulation of the choice conditions. More details are found below in the Methods section.

\section{Methods}

\section{Participants}

The study was approved by the Institution Review Board (IRB) before data collection. Participants were recruited from Amazon's Mechanical Turk (MTurk) under the restriction that they were U.S. residents (based on ownership of a U.S. bank account). To motivate participants, the present study paid $\$ 3.00$ for $30 \mathrm{~min}$, which is relatively generous compared to the median hourly wage of $\$ 1.38$ (Horton and Chilton 2010).

Participants were recruited from MTurk with two separate online surveys. ${ }^{1}$ One survey recruited older adults over the age of 59 while the other survey targeted younger adults whose age was between 18 and 30 . Other than the age requirement stated in the recruitment post, the content of the surveys was identical. To doublecheck that participants met the age requirement, participants were asked to report their age after completing the choice tasks and psychological scales. Those who reported a wrong age (e.g., someone who took the older adults

\footnotetext{
${ }^{1}$ Following some previous studies (e.g., Reed et al. 2008), we employed two generation cohort samples to represent the two age groups. Additionally, the age requirements were based on a pilot study. For more details please refer to the Appendix.
} 
survey but reported an age younger than 59) were removed. One hundred and twenty-four participants took the older adult survey. Eight participants were removed because they reported a wrong age or failed to complete at least $50 \%$ of items. Another one hundred and twenty-eight participants took the younger adults survey. Sixteen participants were removed because they reported a wrong age, failed to report an age, or completed less than 50\% of items. Thus, the effective sample size was one hundred and sixteen for the older adult group, and one hundred and twelve for the younger adult group. For demographic variables please refer to the Results section.

Given that this study involves cognition, some might be concerned with whether some participants, particularly in the older adult group, may have major cognitive impairments such as dementia. We used two approaches to address this concern. First, recruited participants needed to have a high reputation on the platform of MTurk (at least a 95\% approval ratings from their previous HITs - the default setting in MTurk in which many researchers use). Second, in the older adult group, there were four participants who answered all numeracy questions incorrectly. When taking a closer look at these participants' responses, there was no clearly odd response for other questions. Additionally, as reported below, scores on numeracy were comparable between the two age groups. Thus, we believe our sample was not contaminated by significant cognitive impairments.

Sensitivity analysis was performed with $G *$ Power 3.1 .9 to estimate the effect sizes with the current sample size. Statistical power was set at .80 with an $\alpha$ of .05 . As a result, the study had sufficient power to detect a coefficient of determination $\left(R^{2}\right)$ of .07 in a multiple regression with five individual predictors (age differences, gender, education, numeracy, and choice condition) and two double interactions (between age and choice condition, and between numeracy and choice condition; as introduced below). Such a coefficient of determination was smaller than the median level (.13) defined by Cohen (1988).

\section{Materials \& Procedures}

Participants completed web-based questionnaires via Qualtrics, where consumer and gamble choice tasks were given along with a numeracy scale, and a demographic survey, as listed below.

\section{Decision-Making Tasks and Choice Condition Manipulation (Hyperchoice Vs. Simple Choice)}

In the present study, each participant completed two choice tasks: a consumer choice task involving mattresses and a gamble choice task. To test the hypotheses, each choice task had two versions. The first version employed a simple, non-hyperchoice condition, while the second version employed a more complex, hyperchoice condition. Participants were randomly assigned into one of the two versions. The assignment of the simple-choice and hyperchoice conditions remained the same for participants across the two choice tasks. For example, if a participant was assigned a simple-choice condition in the mattress choice task, that meant that participant was also assigned a simple-choice choice condition in the gamble choice task. After completing each choice task, participants were asked to report their experience. As shown in the appendix, there was no significant difference in demographics and numeracy between participants in the hyperchoice condition and participants in the simple-choice condition.

Consumer choice task The products used for consumer decision making were mattresses varying in price and customer rating out of 100 . We chose to use mattresses as the product in the consumer choice task because most Americans believe finding a suitable mattress will improve quality sleep, which is an important aspect of well-being for all ages. For instance, using a sample of 1500 adults ages 25 to 55 , the National Sleep Foundation (2010) reported that "about 9 in 10 adults rated that having a comfortable mattress $(92 \%)$ in getting a good night's sleep is important."

As described, the mattress task included two choice conditions: a hyperchoice condition and a simple-choice condition. In the simple-choice condition, there were four separated trials. In each trial participants were presented with four mattresses and were asked to choose one out of four. In the hyperchoice condition, 16 mattresses were presented simultaneously, and participants were asked to pick four out of 16 mattresses. ${ }^{2}$ The set size between the two choice conditions (four options vs. 16 options) was also adopted in Chernev (2003). Across the four trials, the mattresses were never repeated. In other words, the mattresses in both choice conditions were identical - simply shown in different presentation formats (sixteen simultaneously vs. four per trial). For the mattresses, the prices ranged from $\$ 351$ to $\$ 1089$, with a mean of $\$ 709$ and standard deviation of $\$ 211$. The rating (out of 100) ranged from 32 to 97 , with a mean of 63 and standard deviation of 19.

Gamble choice task After completing the mattress choice task, participants were asked to respond to the gamble choice task. Each gamble choice option gave a probability statement of winning a certain amount of money. Again, participants were randomly assigned into either the hyperchoice condition or the simple-choice condition. Similar to the mattress choice task, in the hyperchoice condition, participants selected four

\footnotetext{
${ }^{2}$ While we understood that selecting four mattresses did not perfectly reflect decisions in everyday life, as discussed earlier, the present study aimed to make characteristics other than amount of information as similar as possible between the choice conditions. Thus, following the simple-choice condition, we asked participants in the hyperchoice condition also to make four choices before reporting their experience. It is worth noting that making trade-offs between internal and external validities are common in psychological research (Morling 2015). On the other hand, selecting multiple options is the gamble choice task mimics the everyday life activity better (e.g., investing multiple stock portfolios).
} 
gambles out of 16 . In the simple-choice condition, there were four trials, and in each trial participants selected one option out of four. The options were identical between the hyperchoice and simple-choice conditions. Across the 16 options, the money ranged from $\$ 87$ to $\$ 578$, with a mean of $\$ 342$ and standard deviation of $\$ 136$. The probability ranged from .11 to .94 , with a mean of .51 and standard deviation of .27 .

Dependent variables: decision satisfaction and decision difficulty As for dependent variables, following the important work in this domain (e.g., Chernev 2003; Iyengar and Lepper 2000; Scheibehenne et al. 2009), the study employed self-report measures. Participants completed two five-point Likert scales after completing each choice task. The first scale asked, "Are you satisfied with your (mattress choice or gamble choice) decisions?" with 1 indicating highly satisfied and 5 indicating highly dissatisfied. The second scale stated, "I found the (mattress or gamble) decision-making task difficult" with 1 representing highly agree and 5 representing highly disagree.

\section{Rasch-Based Numeracy Scale}

Developed by Weller et al. (2013), the Rasch-Based Numeracy Scale consists of eight numeric-entry-type items. Example questions from the numeracy scale include, "Imagine that we roll a fair, six-sided die 1000 times. Out of 1000 rolls, how many times do you think the die would come up as an even number?," and "In the ACME PUBLISHING SWEEPSTAKES, the chance of winning a car is 1 in 1000 . What percent of tickets of ACME PUBLISHING SWEEPSTAKES win a car?" The score for numeracy was computed as the rate of correct responses (number of correct responses out of eight). In the current sample, the Cronbach's $\alpha$ of the numeracy scale was .74.

\section{Demographic Survey}

Participants were asked to report their age with an open-ended question. Gender was coded with 1 as male and 2 as female. Race was measured with six options: 1: White; 2: Black or African American; 3: American Indian or Alaska Native; 4: Asian; 5: Native Hawaiian or Pacific Islander; and 6: Other. Education was coded by six levels in order: 1: high school graduate (high school diploma or equivalent including GED); 2: some college but no degree; 3 : Associate's degree in college (2-year); 4: Bachelor's degree in college (4-year); 5: Master's degree; 6: Doctoral degree or Professional degree (JD, MD).

\section{Data Analysis Approach}

SPSS 24.0 was used for data analyses. Descriptive statistics were first reported to show demographic variables and performance on numeracy. To test the main effect of hyperchoice, as well as the interactions between hyperchoice and age differences and numeracy, four multiple regressions were performed, with decision difficulty or decision satisfaction in one choice task as the outcome variable, and age differences, gender, education, choice condition (hyperchoice vs. simplechoice), numeracy, interaction between choice condition and age differences, and interaction between choice condition and numeracy as the predictors. To avoid potential multicollinearity led by the interaction terms, before performing the regressions, the variables of age differences and numeracy were centered with their means. The variable of choice condition (hyperchoice vs. simple-choice) was dummy coded, with simple-choice condition as the reference level. In all four regressions, the smallest observed Tolerance was .43, which was greater than the cut-off values of .1 or .2 as proposed by other researchers (Belsley et al. 1980; Hair et al. 2010). Therefore, multicollinearity did not appear to be an issue in our analyses.

For the predictor of age differences, it is worth noting that there was little difference in statistical significance or conclusion between treating age differences with two categories $(0=$ older adult group, 1 = younger adult group) and treating age differences with all collected values (a variety of numerical values collected from participants). However, we chose to treat the variable of age differences with all collected values for two reasons. First, treating age differences with just two categories ignored the individual differences in age within each of the group. Second, when using age differences with the collected values, the $R^{2}$ (as reported in Table 2) was higher in all models than when using age differences with two categories. The aforementioned approach of treating age as a continuous variable in regression was also adopted in Finucane and Gullion (2010).

As reported below, the interaction between numeracy and choice condition was significant on decision difficulty and decision satisfaction in the gamble choice task. To unpack the interaction, we divided participants into a low numeracy group (correct response rate $\leq .625,47.8 \%$ of participants) and a high numeracy group (correct response rate $>.625,52.2 \%$ of participants). In our sample, using the correct response rate of .625 as the cut-off value was similar to using median split to create two groups. We did not use the regular approach of mean \pm 1 SD to create groups because numeracy was not normally distributed (see the Results section). ${ }^{3}$ It is worth noting that the approach of using median split to create a higher and a lower numeracy group was also adopted in other studies on numeracy (Peters et al. 2009; Peters et al. 2014; Peters and Bjalkebring 2015).

\footnotetext{
${ }^{3}$ With the original numeracy data, the skewness was -.73 , which was at a moderate skew level. However, the skewness increased to -1.01 after applying the natural-log transformation. Consistently, the Q-Q plots of numeracy deviated from the diagonal line to a larger extent after using the natural-log transformation. Previous research also indicated that log-transformation might increase skewness in some cases (Feng et al. 2014). Therefore, the current study adopted the original values rather than the transformed values of numeracy as the predictor when running regression analyses.
} 
Table 1 Demographic variables between the two groups

\begin{tabular}{llllll}
\hline & Age range & Mean Age (SD) & $\begin{array}{l}\text { Females to } \\
\text { Males Ratio }\end{array}$ & $\begin{array}{l}\text { Mean Education } \\
\text { Level (SD) }\end{array}$ & Ethnicity/Race \\
\hline Older adults & $59-96$ & $67.88(9.06)$ & 69 to 47 & $4.14(1.53)$ & $\begin{array}{c}\text { 105 White, 5 Black or African American, 2 Asian } \\
\text { American, 4 other or multiracial }\end{array}$ \\
Younger adults & $18-29$ & $23.97(2.53)$ & 53 to 59 & $3.83(1.17)$ & $\begin{array}{c}70 \text { White, 18 Black or African American, 12 Asian } \\
\text { American, 1 Native American, 11 other or multiracial }\end{array}$ \\
\hline
\end{tabular}

\section{Results}

\section{Demographic Variables}

Table 1 shows the demographic variables between the two groups. There was a significant difference in age between the two groups, $t(226)=49.47, p<.001$, Cohen's $d=6.58$. The difference in education between the two groups was at a marginally significant level, $t(226)=1.77, p=.078$, Cohen's $d=0.24$, probably because some younger adults have not had sufficient time to complete their degrees. The younger adult group appeared to be slightly more racially diverse in race than the older adult group.

\section{Numeracy}

For numeracy, its score was the rate of correct responses. Figure 1 (a-c) displays the distribution of the rate for all participants, older adults and younger adults. As can be seen, in each figure, fewer than $5 \%$ of participants answered all questions incorrectly or correctly. Thus, the current sample did not face an obvious floor or ceiling effect. Additionally, the distribution in each figure was somewhat negatively skewed, with about half of participants answering six or seven items correctly and another half answering five items or fewer incorrectly. The difference between the two age groups did not reach a strict significance level, Mann-Whitney $U=1.78$, $p=.075$, median numeracy in older adults $=.63(\mathrm{IQR}=.47)$, median numeracy in younger adults $=.75(\mathrm{IQR}=.38){ }^{4}$

\section{Effects of Age Differences, Numeracy and Choice Condition on Decision Difficulty and Decision Satisfaction in the Gamble Choice Task}

In the gamble choice task, after making their decisions, participants reported (1) how much difficulty they experienced when making those decisions (a smaller number represented greater experienced difficulty), and (2) whether they were satisfied with their decisions (a smaller number represented greater decision satisfaction). Across the participants, the mean (SD) of decision difficulty and decision satisfaction in

\footnotetext{
${ }^{4}$ Using independent $t$-test did not change the significance of the result.
}

the gamble choice task were 3.46 (1.21) and $1.96(0.81)$, respectively. Within the task, greater experienced difficulty was associated with lower satisfaction, implying good validity of our measures: $r(225)=-.46, p<.001$ in the gamble choice task. Tables 2 illustrates the effects of demographic variables, numeracy and choice condition on decision difficulty and decision satisfaction in the gamble choice task.

As shown in Table 2, in the gamble choice task, older individuals reported greater decision difficulty than younger individuals. Moreover, older adults also were less satisfied with their decision. Unlike our predictions, age differences did not interact with choice condition (hyperchoice vs. simple choice). Hence, age was generally negatively related to decision experience in the gamble choice task.

In terms of the effect of choice condition, regarding decision difficulty, the main effect of choice condition was significant, indicating that hyperchoice condition was generally associated with greater decision difficulty. Furthermore, choice condition also interacted with numeracy on both decision difficulty and decision satisfaction in the gamble choice task. Following the approach of unpacking the interaction as stated above, Figs. 2 and 3 depict the interactions between numeracy and choice condition. As shown in Fig. 2, in the gamble choice task, the variation in experiencing difficulty between the two choice conditions was smaller in higher numerate participants than in lower numerate participants. A contrast analysis confirmed this: $F(1,224)=5.36, p=.020$. In other words, higher numerate participants experienced a similar level of difficulty $(F(1,224)=0.001, p=.978)$ whereas lower numerate participants experienced more difficulty $(F(1,224)=$ $10.41, p=.001)$ in the hyperchoice condition than in the simple-choice condition.

As shown in Fig. 3, in the gamble choice task, a contrast analysis indicated that higher numerate participants and lower numerate participants displayed different trends of satisfaction between the hyperchoice and simple-choice conditions, $F(1,224)=6.85, p=.009$. More specifically, higher numerate participants had relatively stable satisfaction between the hyperchoice and simple-choice conditions, $F(1,224)=1.85, p=.175$. By contrast, lower numerate people were more dissatisfied in the hyperchoice condition than in the simple-choice condition, $F(1,224)=5.37, p=.021$. 
Table 2 Effects of demographic variables, numeracy and choice condition on decision difficulty and decision satisfaction in the gamble choice task

\begin{tabular}{|c|c|c|c|c|}
\hline Outcome variable & Predictors & $\mathrm{B}(\mathrm{SE})$ & $R^{2}$ & $F$ \\
\hline \multirow[t]{8}{*}{ Difficulty } & & & .17 & $6.33, p<.001$ \\
\hline & Age differences & $-.01(.005), p=.006$ & & \\
\hline & Education & $.01(.06), p=.868$ & & \\
\hline & Gender & $.24(.16), p=.120$ & & \\
\hline & Numeracy & $.55(.47), p=.247$ & & \\
\hline & Choice condition & $-.31(.15), p=.037$ & & \\
\hline & Choice condition * Age differences & $.001(.007), p=.892$ & & \\
\hline & Choice condition $*$ Numeracy & $1.24(.63), p=.050$ & & \\
\hline \multirow[t]{8}{*}{ Satisfaction } & & & .08 & $2.60, p=.013$ \\
\hline & Age differences & $.01(.003), p=.004$ & & \\
\hline & Education & $.02(.04), p=.711$ & & \\
\hline & Gender & $.15(.11), p=.193$ & & \\
\hline & Numeracy & $.52(.34), p=.124$ & & \\
\hline & Choice condition & $.07(.11), p=.494$ & & \\
\hline & Choice condition * Age differences & $-.007(.005), p=.120$ & & \\
\hline & Choice condition $*$ Numeracy & $-1.14(.45), p=.011$ & & \\
\hline
\end{tabular}

Decision difficulty: a smaller value indicated greater decision difficulty.

Decision satisfaction: a smaller value indicated greater satisfaction.

Choice condition: dummy coded with simple-choice $=0$ and hyperchoice $=1$.

Gender: male $=1$ and female $=2$.

\section{Effects of Age Differences, Numeracy and Choice Condition on Decision Difficulty and Decision Satisfaction in the Consumer Choice Task}

In the mattress choice task, the mean (SD) of decision difficulty and decision satisfaction was 3.39(1.16) and 2.06(0.81), respectively. Similar to the gamble choice task, there was a significant relationship between decision difficulty and decision satisfaction, $r(226)=-.39, p<.001$.

Table 3 presents the regression results on decision experience in the mattress choice task. As for the effect of age differences, there were two similarities between the gamble and mattress choice tasks. First, age was negatively related to experienced difficulty. Second, there was no significant interaction between age differences and choice condition. Thus, the effect of age differences were relatively consistent in our study. For the effect of choice condition, similar to the gamble choice task, we found participants experienced more difficulty in the hyperchoice condition than in the simple-choice condition in the mattress choice task. However, unlike the gamble choice task, there was no interaction between numeracy and choice condition for choosing mattresses. A possible reason might be that numerical information was more abstract (e.g., probability) in the gamble choice task than in the mattress choice task, and hence, demanded greater involvement of numeracy in the gamble task, particularly in the hyperchoice condition.

\section{Discussion}

With the rise of the number of options people have to choose from nowadays, the hyperchoice decision-making process has become common. The present study expanded the findings of hyperchoice and decision experience in three domains. First, while past studies on hyperchoice overwhelmingly focused on consumer choice, the current study further extended the research to the gamble choice field. Second, our work exhibited a moderation effect on hyperchoice. Hence, we not only provided support to the conclusions of the past meta-analysis studies (Chernev et al. 2015; Scheibehenne et al. 2010) but also shed more light on how hyperchoice specifically affected decision experience. Third, research on numeracy has more recently gained popularity because it is critical to decision making and a variety of other cognitive functions (Ghazal et al. 2018). The present study advanced the knowledge of both numeracy and hyperchoice by building a novel connection between the two. Below we discussed our main findings, implications, and limitations, respectively.

\section{Main Findings}

Overall, the present study found hyperchoice either had a main effect or interacted with numeracy on decision experience. First, in both gamble and consumer choice tasks, hyperchoice was positively associated with decision 

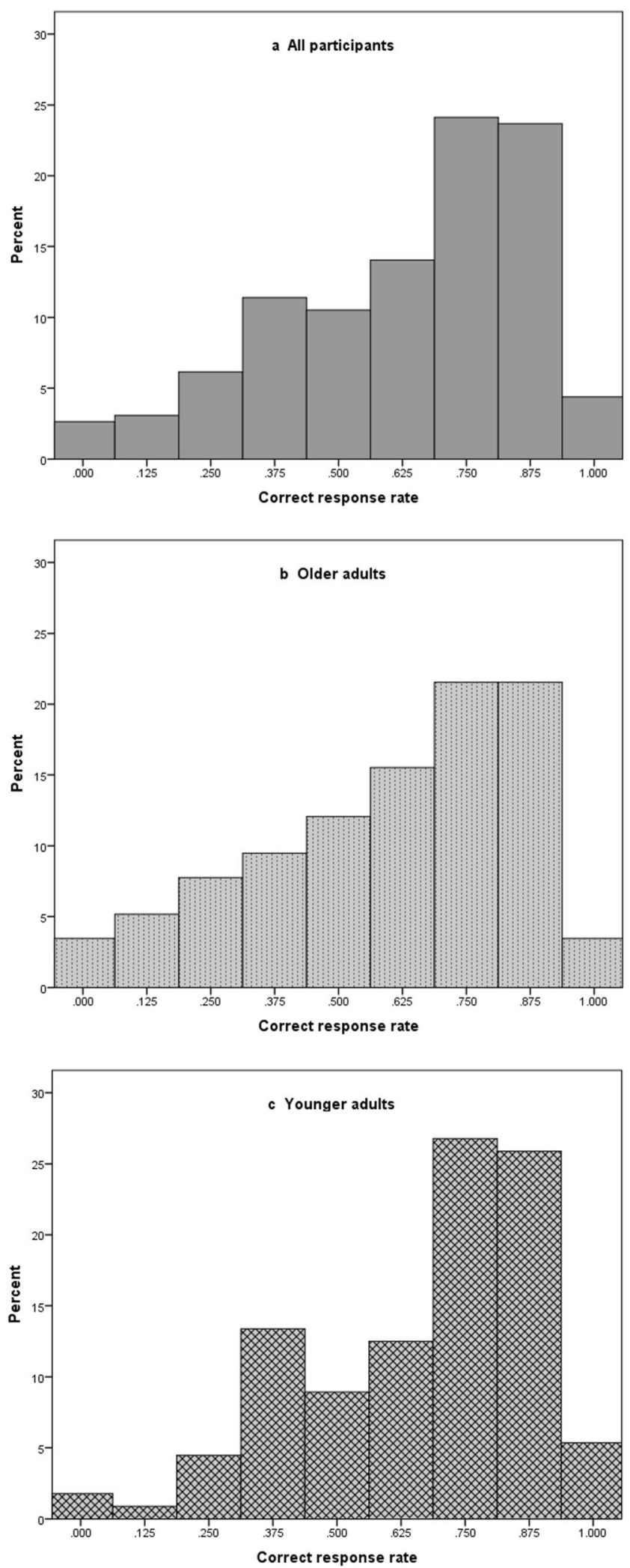

Fig. 1 (a-c). Distribution of numeracy correct response rate

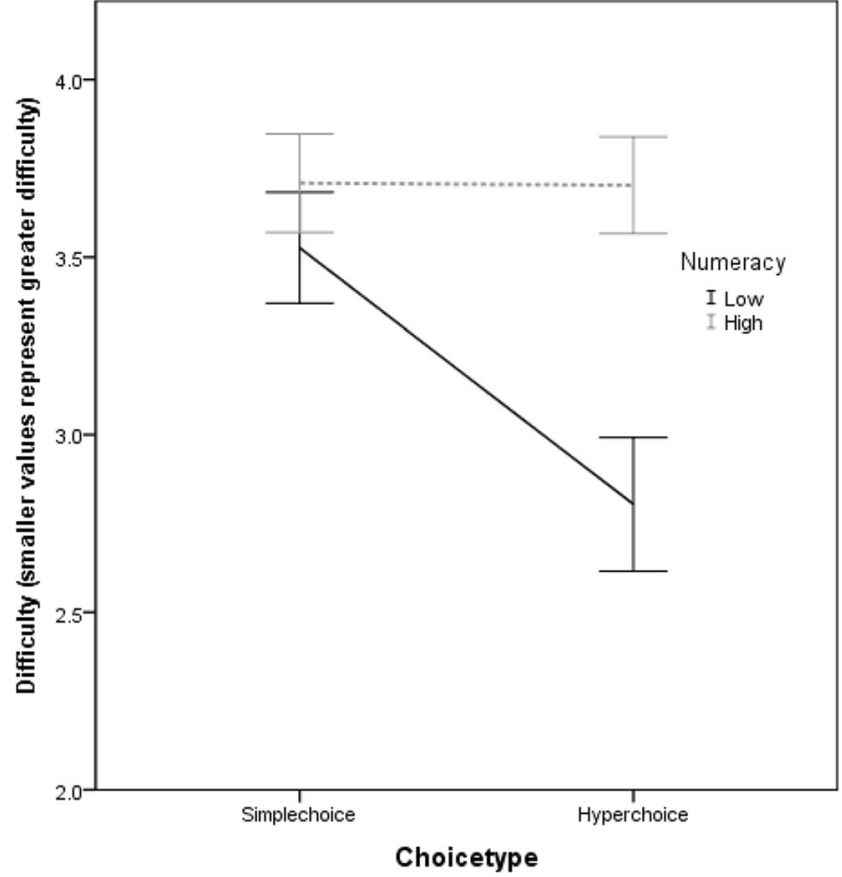

Fig. 2 Interaction between choice condition and numeracy in decision difficulty in the gamble choice task

difficulty, indicating that increasing the number of options was related to more experienced difficulty.

Second, beyond the main effect, the function of hyperchoice was complex in the gamble choice task due to its interaction with numeracy. As depicted in Fig. 2, whereas higher numerate people's experienced decision difficulty remained relatively constant between the hyperchoice and

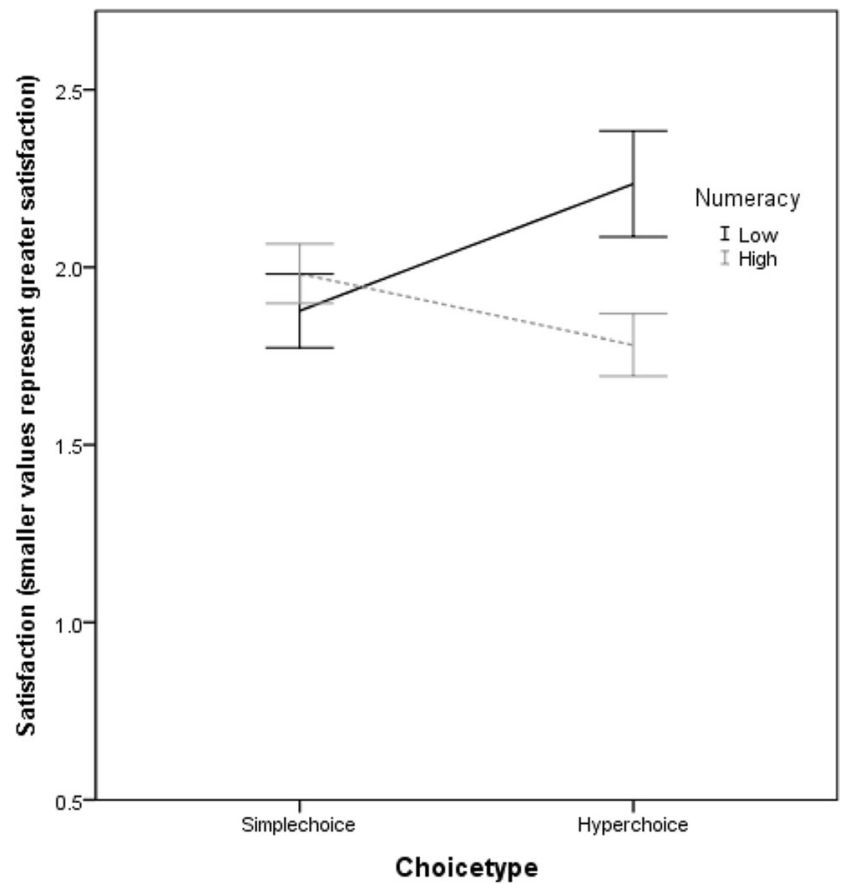

Fig. 3 Interaction between choice condition and numeracy in decision satisfaction in the gamble choice task 
Table 3 Effects of demographic variables, numeracy and choice condition on decision difficulty and decision satisfaction in the consumer choice task

\begin{tabular}{|c|c|c|c|c|}
\hline Outcome variable & Predictors & $B(S E)$ & $R^{2}$ & $F$ \\
\hline \multirow[t]{8}{*}{ Difficulty } & & & .13 & $4.49, p<.001$ \\
\hline & Age differences & $-.009(.005), p=.049$ & & \\
\hline & Education & $-.03(.06), p=.638$ & & \\
\hline & Gender & $-.08(.15), p=.584$ & & \\
\hline & Numeracy & $.61(.47), p=.191$ & & \\
\hline & Choice condition & $-.41(.15), p=.006$ & & \\
\hline & Choice condition $*$ Age differences & $-.001(.007), p=.851$ & & \\
\hline & Choice condition * Numeracy & $.49(.62), p=.431$ & & \\
\hline \multirow[t]{8}{*}{ Satisfaction } & & & .06 & $1.98, p=.060$ \\
\hline & Age differences & $.001(.003), p=.828$ & & \\
\hline & Education & $.10(.04), p=.011$ & & \\
\hline & Gender & $.27(.11), p=.016$ & & \\
\hline & Numeracy & $.36(.34), p=.290$ & & \\
\hline & Choice condition & $.04(.11), p=.745$ & & \\
\hline & Choice condition * Age differences & $-.002(.005), p=.740$ & & \\
\hline & Choice condition $*$ Numeracy & $.05(.45), p=.917$ & & \\
\hline
\end{tabular}

Decision difficulty: a smaller value indicated greater decision difficulty.

Decision satisfaction: a smaller value indicated greater satisfaction.

Choice condition: dummy coded with simple-choice $=0$ and hyperchoice $=1$.

Gender: male $=1$ and female $=2$. simple-choice conditions, lower numerate people felt much more difficulty in the hyperchoice condition than in the simple-choice condition. Additionally, as shown in Fig. 3, lower numerate people were less satisfied with their decision in the hyperchoice condition than in the simple-choice condition. By contrast, higher numerate people's satisfaction was stable between the two choice conditions, probably because they had sufficient ability to seek satisfying options in both choice conditions. Thus, the findings implied that lower numerate people were more sensitive to the challenges imposed by hyperchoice. When there were more options to weigh in, lower numerate people faced more difficulty and were less satisfied.

Third, unlike the gamble choice task, hyperchoice did not interact with numeracy in the consumer choice task. Numeracy did not have a significant main effect in this task either. We believe the difference in the function of numeracy between the gamble choice task and the consumer choice task was because the gamble choice task was more complex in numerical information. For example, compared to price and customer rating, probability was more abstract and difficult to comprehend. Thus, with a list of probabilities, lower numerate people might face some extra challenges in the hyperchoice condition in the gamble choice task. Hence, the specific effect of hyperchoice may also depend on the type of choice tasks.

Fourth, compared to younger adults, older adults had greater decision difficulty in both tasks, and lower decision satisfaction in the gamble choice task. Notably, the variable of age differences did not interact with hyperchoice or numeracy, even though we purposely widened the age gap between the two groups when recruiting participants. Thus, the findings suggested that the impacts of hyperchoice and numeracy on decision experience was similar between younger adults and older adults. While our study found older adults generally had more negative decision experience in both choice conditions in the gamble and consumer choice tasks, the reasons were not immediately clear. There are some possible interpretations. First, declined cognitive capacity and mental and physical energy could make it more difficult for older adults to go through and analyze options, even when the number of options is limited. Second, older adults have more life experiences and knowledge and as a result, they may have higher expectations. Thus, regardless of how many options are being presented to them, they might believe there is always a better option somewhere, making them less likely to be satisfied. Third, socioemotional selectivity theory posits that because older adults increasingly recognize that they have limited time left to live, they become more motivated to optimize positive emotional experiences in the "here and now" (Carstensen, 2006). As there is no dominant option and trade-offs have to be made in both choice conditions, older adults may feel the choices are difficult to make to optimize their experience, and hence, are not satisfied. From another perspective, younger adults may feel they have more time and opportunities. Thus, they are less likely to be stressed when making decisions. Fourth, given that older adults have higher expectations 
and motivation to seek emotionally meaningful experience, maybe choice options used in the present study or the overall experience of taking this survey were not "meaningful; therefore, they were not satisfied. Similarly, older adults might be more likely to make repeat decisions. Thus, when faced with making a new decision or not given options that they have tried before, older adults might be less likely to be satisfied as well as find the decision-making process more difficult. However, we are mindful that the exact mechanisms underlying the relationship between age and decision experience demand further research.

\section{Implications}

The present study generated several implications. First, while most past research employed consumer choice tasks to examine the hyperchoice effect, the present study demonstrated that such an effect could be extended to a gamble choice task. Our findings indicated the effect of hyperchoice (and its interaction with other factors) may be common in the field of judgement and decision making. Future studies can further examine how hyperchoice affects other decision tasks such as intertemporal choice and moral dilemma tasks.

Second, from a practical perspective, the study implied that merchants should try to ease the process of decision making in the presence of hyperchoice. There are a few strategies to consider. One strategy, as adopted by the wholesale giant Costco or the global discount supermarket ALDI, is to limit product selection. This strategy was echoed in a study on holiday destination choice (Thai and Yuksel 2017). The authors suggested travel agencies use a screening tool to narrow down the range of options and present a customized portfolio. Similarly, for wedding dress consultants, their goal is to narrow wedding dress options down based on the bride's preference to find the "perfect" dress. Another strategy is related to the presentation format of the options. In the present study, participants in both hyperchoice and simple-choice conditions faced same options. The difference was that options in the hyperchoice condition were presented altogether whereas options in the simple conditions were presented in separated groups. Thus, to improve decision experience, merchants can exhibit goods in groups and sequentially rather than in a holistic cluster. Additionally, Hoch, Bradlow, \& Wansink (1999) showed customers were more satisfied when options were displayed in an organized than a random manner. Hence, online merchants should strengthen the filter function so that consumers can rank the items based on their preference. For example, for apartment searching, units can be sorted by the number of bedrooms, number of bathrooms, price, pet allowance, garage/parking availability and other factors.

Third, the study further highlighted the importance of numeracy in decision making. Past studies have found low numeracy is associated with inferior decision outcomes (Cheng
2020; Reyna et al. 2009; Sinayev and Peters 2015). The present study extended the findings to decision experience and found lower numerate people faced extra challenges in the scenario of hyperchoice. Hyperchoice gains popularity nowadays, and people need to deal with abstract and complex numerical information commonly (e.g., build an investment portfolio for retirement). Thus, the study advocated for math and numeracy education (Gravemeijer et al., 2017), especially in countries with "math crisis" (Anderson, 2016). Some recent studies found game-based learning and learning via virtual social networks could increase students' motivation in learning (Partovi and Razavi 2019; Razavi 2018; Sung et al. 2012). Thus, from a practical perspective, it may be beneficial for schools to incorporate such techniques in numeracy learning.

\section{Limitations}

This study has some limitations that should be acknowledged. First, one main goal of the present study was to examine how older adults experience hyperchoice scenarios in the era of information explosion. Hence, following some previous studies as cited earlier, we employed an older adult group and a younger adult group. While this design allowed us to describe decision experience in the older adults by making comparisons between the two age groups, one limitation was that middle-aged adults were not tested. Thus, our study did not capture the decision experience from a full developmental perspective, meaning comparisons between younger and older age groups may not reveal the complete developmental trajectories of decision making across the lifespan. Moreover, our study did not depict the consumer behavioral pattern comprehensively as the middle-aged persons are the main consumers in the society. To address these issues, future research should include participants with a wide range of ages (or break age into smaller life stage groups) to better reveal the effect of age on decision making in a variety of choice conditions.

Second, our sample was not particularly racial/ethnically diverse; consequently, our study was not informative about the relationship between race/ethnicity and hyperchoice. As diversity gains its importance in the current society, investigating the relationship between race/ethnicity and decision making would definitely add to the literature.

In addition, following previous studies (e.g., Iyengar and Lepper 2000; Lee 2017; Scheibehenne et al. 2009, 2010), the current work employed self-report measures to index subjective decision experience. While such measures are informative, people may have different interpretations of the numerical values on the Likert scale. Future research could further adopt dynamic objective measures such as eye-tracking and mouse-tracking (e.g., Cheng and González-Vallejo 2017; Reeck et al. 2017) to depict decision experience in the scenario of hyperchoice.

Lastly, the present study manipulated the hyperchoice condition with 16 options. While 16 options went beyond binary 
choices employed in many studies, the number might still be smaller than what people actually experience in everyday life (the Amazon and Medicare examples described in the Introduction). To enhance external validity, future studies may further increase the number of options to examine how people make decisions. Similarly, while the present study employed consumer and gamble choice tasks, it is possible that the effects of hyperchoice could be extended to more consequential decisions such as health-care choices and larger financial investment decisions (e.g., buying a house).

\section{Conclusion}

The present study suggests that hyperchoice conditions generally impose greater challenges in participants. Furthermore, its specific effect might be moderated by individual characteristics and decision task type. Therefore, beyond the main effect, future studies on hyperchoice should take contextual and personal factors into account to better understand how hyperchoice affects decision making. Our findings also advocate for numeracy education and strategy development to ease decision experience in the scenario of hyperchoice.

\section{Compliance with Ethical Standards}

Conflict of Interest On behalf of all authors, the corresponding author states that there is no conflict of interest.

Ethical Statement All procedures performed in studies involving human participants were in accordance with the ethical standards of the institutional review board (IRB 18-0193) at the University of Northern Iowa with the 1964 Helsinki declaration and its later amendments or comparable ethical standards.

Informed Consent Informed consent was obtained from all individual participants included in the study.

\section{Appendix}

\section{Age requirements}

The age ranges in the present work (18-30 for younger adults and over 59 for older adults) were based on a pilot study in which we tested the study materials in older adults in a senior care facility and younger adults in a college. The purpose of the pilot study was to make sure the study materials made sense to participants. In the pilot study, the age ranges were 18-30 and over 59 for younger and older adults, respectively. Hence, in the formal study, we continued using the same age ranges to recruit participants.

It is worth noting that the distinct age requirements between the two groups were arbitrary; however, we did so for two reasons. First, the present study collected data from MTurk.
One potential limitation of MTurk is that there are more younger adults than older adults (Hitlin 2016). Consistently, in Lee's (2017) study about hyperchoice, more than $80 \%$ of participants recruited from MTurk were below 30 , and only $6.5 \%$ were above 50 . Thus, in order to ensure the study had sufficient older adult participants and to detect the possible psychological experience in them, we purposely separated older adults and younger adults with two independent surveys.

Second, some previous studies examining the effect of age differences on decision making and other cognitive functions (e.g., working memory) adopted two generation cohort samples (i.e., two independent age groups). More specifically, in one study testing the effect of aging and choice set size on decision making, the ages for the younger adult group and the older adult group were 18-24 and 60-94, respectively (Reed et al. 2008). Similarly, in a study examining the relationship of age differences and attitude toward risk, the ages for the younger and older participant groups were 16-23 and 63-87, respectively (Rolison et al. 2012). In the field of working memory, a study on effect of aging and reward anticipation employed two age groups with 20-27 for the younger adults and 65-78 for the older adults (Thurm et al. 2018). One goal of the current work was to test older adults' decision experience in the scenarios with different choice set sizes. Therefore, following these studies, we employed two generation cohort groups that allowed us to compare younger adults and older adults.

\section{Demographics and numeracy comparisons between the two choice conditions}

Independent $t$-tests were performed in age and education between those in the hyperchoice condition and those in the simple-choice condition. As a result, there was no significant difference in age, $t(226)=.14, p=.887$, mean $_{\text {hyperchoice }}=$ 46.10, mean $_{\text {simple choice }}=46.53$. Similarly, there was no significant difference in education, $t(226)=.24, p=.810$, meanhyperchoice $=3.97$, mean $_{\text {simple choice }}=4.01$.

A chi-square test was conducted to test if there was an association between gender and choice condition. Consequently, the test did not reach the statistical significance level, chi-square $=3.01, p=.083$, indicating gender was equally distributed between the two choice conditions.

Additionally, as indicated in the main text, numeracy was not significantly different between the two choice conditions.

An independent $t$-test was conducted to compare numeracy between the two choice conditions. There was no significant difference in numeracy between hyperchoice condition $($ mean $=.65)$ and simple-choice condition $($ mean $=.62)$, $t(226)=.51, p=.483$. 


\section{References}

Belsley, D. A., Kuh, E., \& Welsch, R. E. (1980). Regression diagnostics: Identifying influential data and sources of collinearity. New York: Wiley.

Carpenter, S. M., \& Yoon, C. (2012). Aging and consumer decision making. Annals of the New York Academy of Sciences, 1235, E1E12. https://doi.org/10.1016/B978-0-12-417148-0.00017-0.

Chen, Y., \& Sun, Y. (2011). Age differences in financial decision-making: Using simple heuristics. Educational Gerontology, 29(7), 627635. https://doi.org/10.1080/713844418.

Cheng, J. (2020). The role of numeracy and impulsivity in Intertemporal choice and decision making. Psychological Thought, 13(1), 254 272

Cheng, J., \& González-Vallejo, C. (2017). Action dynamics in Intertemporal choice reveal different facets of psychology states. Journal of Behavioral Decision Making., 30(1), 107-122.

Chernev, A. (2003). When more is less and less is more: The role of ideal point availability and assortment in consumer choice. Journal of Consumer Research, 30(2), 170-183. https://doi.org/10.1086/ 376808 .

Chernev, A., Bockenholt, U., \& Goodman, J. (2015). Choice overload: A conceptual review and meta-analysis. Journal of Consumer Psychology, 25, 333-358.

Cohen, J. (1988). Statistical power analysis for the behavioral sciences (Second ed.). Hillsdale, NJ: Lawrence Erlbaum Associates, Publishers.

Cokely, E.T., Feltz, A., Ghazal, S., Allan, J.N., Petrova, D., \& GarciaRetamero, R. (2018). Decision making skill: From intelligence to numeracy and expertise. In K. A. Ericsson, R. R. Hoffman, A. Kozbelt, \& A. M. Williams (2nd Eds.), Cambridge handbook of expertise and expert performance. New York: Cambridge University Press.

Cole, C. A., \& Gaeth, G. J. (1990). Cognitive and age-related differences in the ability to use nutritional information in a complex environment. Journal of Marketing Research, 27, 175-184. https://doi.org/ $10.1177 / 002224379002700205$.

D'Angelo, J., \& Toma, C. (2017). There are plenty of fish in the sea: The effects of choice overload and reversibility on online daters' satisfaction with selected partners. Media Psychology, 20(1), 1-27.

Dror, I. E., Katona, M., \& Mungur, K. (1998). Age differences in decision making: To take a risk or not? Gerontology, 44, 67-71. https://doi. org/10.1159/000021986

Feng, C., Hongyue, W., Lu, N., Chen, T., He, H., Lu, Y., \& Tu, X. (2014). Log-transformation and its implications for data analysis. Shanghai Archives of Psychiatry, 26, 105-109. https://doi.org/10. 3969/j.issn.1002-0829.2014.02.009.

Gluck, M. A., Mercado, E., \& Myers, C. E. (2016). Learning and memory: From brain to behavior. 2nd ed., Macmillan Education.

Gourville, T., \& Soman, D. (2005). Overchoice and assortment type: When and why variety backfires. Marketing Science, 24(3), 382395. https://doi.org/10.1287/mksc.1040.0109.

Hair, J. F., Black, W. C., Babin, B. J., \& Anderson, R. E. (2010). Multivariate Data Analysis (Seventh ed.). Upper Saddle River, New Jersey: Prentice Hall.

Harada, C. N., Natelson Love, M. C., \& Triebel, K. L. (2013). Normal cognitive aging. Clinics in Geriatric Medicine, 29(4), 737-752. https://doi.org/10.1016/j.cger.2013.07.002.

He, W., Goodkind, D., \& Kowal, P. (2016). U.S. Census Bureau, International Population Reports, P95/16-1, An Aging World: 2015, U.S. government Publishing office, Washington, DC.

Hitlin, P. (2016). Turkers in this canvassing: Young, well-educated and frequent users. Pew research center. Retrieved from https://www. pewinternet.org/2016/07/11/turkers-in-this-canvassing-young-welleducated-and-frequent-users/
Horton, J. J., \& Chilton, L. B. (2010). The labor economics of paid crowdsourcing. In Proceedings from EC '10: The 11th ACM conference on electronic commerce (pp. 209-218). New York, NY: ACM. doi:https://doi.org/10.1145/1807342.1807376.

Iyengar, S. S., \& Lepper, M. R. (2000). When choice is demotivating: Can one desire too much of a good thing? Journal of Personality and Social Psychology, 79(6), 995-1006. https://doi.org/10.1037/ 0022-3514.79.6.995.

Jasper, J. D., Bhattacharya, C., \& Corser, R. (2017). Numeracy predicts more effortful and elaborative search strategies in a complex risky choice context: A process tracing approach. Journal of Behavioral Decision Making, 30(2), 224-235.

Jilke, S., Van Ryzin, G., \& Van de Walle, S. (2016). Responses to decline in Marketized public services: An experimental evaluation of choice-overload. Journal of Public Administration Research and Theory, 26(3), 421-432.

Kaiser Family Foundation (2006). Summary/Chartpack: National Survey of Pharmacists and National Survey of Physicians, Findings on Medicare Part D. Retrieved from https://www.kff.org/medicare/ poll-finding/summarychartpack-national-survey-of-pharmacistsand-national/

Larceneux, F., Rieunier, S., \& Fady, A. (2007). The effect of hyperchoice on the consumer and the moderating effect of the brand: An application in the jewelry market. Recherche et Applications en Marketing (English Edition). 22. 43-56. doi:https://doi.org/10. 1177/205157070702200402.

Lee, H. K. (2017). Choice satisfaction under choice overload: The role of consumers' need for variety and product familiarity. International Journal of Costume and Fashion, 17(2), 15-29. https://doi.org/10. 7233/ijcf.2017.17.2.015.

Mick, D. G., Broniarczyk, S. M., \& Haidt, J. (2004). Choose, choose, choose, choose, choose, choose, choose: Emerging and prospective research on the deleterious effects of living in consumer hyperchoice. Journal of Business Ethics, 52(2), 207-211. https:// doi.org/10.1023/B:BUSI.0000035906.74034.d4.

Morling, B. (2015). Research methods in psychology (2nd ed.). New York, NY: W. W. Norton \& Company.

National Sleep Foundation. (2010). Bedroom poll summary of finding. Crofton: WBA Research.

Partovi, T., \& Razavi, M. R. (2019). The effect of game-based learning on academic achievement motivation of elementary school students. Learning and Motivation, 68, 101592.

Payne, J. W., Bettman, J. R., \& Johnson, E. J. (1993). The use of multiple strategies in judgment and choice. In N. J. Castellan Jr. (Ed.), Individual and group decision making: Current issues (pp. 1939). Inc: Lawrence Erlbaum Associates.

Peters, E., \& Bjalkebring, P. (2015). Multiple numeric competencies: When a number is not just a number. Journal of Personality and Social Psychology, 108(5), 802-822. https://doi.org/10.1037/ pspp0000019.

Peters, E., Dieckmann, N. F., Västfjäll, D., Mertz, C. K., Slovic, P., \& Hibbard, J. H. (2009). Bringing meaning to numbers: The impact of evaluative categories on decisions. Journal of Experimental Psychology: Applied, 15(3), 213-227. https://doi.org/10.1037/ a0016978.

Peters, E., Sol Hart, P., Tusler, M., \& Fraenkel, L. (2014). Numbers matter to informed patient choices: A randomized design across age and numeracy levels. Medical Decision Making, 34(4), 430 442. https://doi.org/10.1177/0272989X13511705.

Razavi, M. R. (2018). Gender differences in the effect of virtual social networks use on students' academic performance. Current Psychology, 1-7. https://doi.org/10.1007/s12144-018-9991-7.

Reeck, C., Wall, D., \& Johnson, E. J. (2017). Search predicts and changes patience in intertemporal choice. Proceedings. National Academy of Sciences. United States of America, 114, 11890-11895. https://doi. org/10.1073/pnas.1707040114. 
Reed, A. E., Mikels, J. A., \& Simon, K. I. (2008). Older adults prefer less choice than younger adults. Psychology and Aging, 23(3), 671-675. https://doi.org/10.1037/a0012772.

Reyna, V. F., Nelson, W. L., Han, P. K., \& Dieckmann, N. F. (2009). How numeracy influences risk comprehension and medical decision making. Psychological Bulletin, 135(6), 943-973. https://doi.org/ 10.1037/a0017327.

Rolison, J. J., Hanoch, Y., \& Wood, S. (2012). Risky decision making in younger and older adults: The role of learning. Psychology and Aging, 27(1), 129-140. https://doi.org/10.1037/a0024689.

Scheibehenne, B., Greifeneder, R., \& Todd, P. (2009). What moderates the too-much-choice effect? Psychology and Marketing, 26(3), 229253. https://doi.org/10.1002/mar.20271.

Scheibehenne, B., Greifeneder, R., \& Todd, P. (2010). Can there ever be too many options? A meta-analytic review of choice overload. Journal of Consumer Research, 37(3), 409-425. https://doi.org/10. 1086/651235.

Sinayev, A., \& Peters, E. (2015). Cognitive reflection vs. calculation in decision making. Frontiers in Psychology, 6, 1-16. https://doi.org/ 10.3389/fpsyg.2015.00532.

Sung, H. Y., Hwang, G. J., Hung, C. M., \& Huang, I. W. (2012). Effect of learning styles on students' motivation and learning achievement in digital game-based learning. 2012 III international conference on advanced applied informatics, 20-22.
Tanius, B. E., Wood, S., Hanoch, Y., \& Rice, T. (2009). Aging and choice: Applications to Medicare part 500. Judgment and Decision making, 4(1), 92-101.

Thai, N., \& Yuksel, U. (2017). Choice overload in holiday destination choices. International journal of culture. Tourism and Hospitality Research, 11(1), 53-66.

Thurm, F., Zink, N., \& Li, S. C. (2018). Comparing effects of reward anticipation on working memory in younger and older adults. Frontiers in Psychology, 9, 2318. https://doi.org/10.3389/fpsyg. 2018.02318 .

Weller, J. A., Dieckmann, N. F., Tusler, M., Mertz, C. K., Burns, W. J., \& Peters, E. (2013). Development and testing of an abbreviated numeracy scale: A Rasch analysis approach. Journal of Behavioral Decision Making, 26, 198-212. https://doi.org/10.1002/bdm.1751.

You, X., Ju, C., Wang, M., Zhang, B., \& Liu, P. (2019). Age differences in the influence of induced negative emotion on decision-making: The role of emotion regulation. The Journals of Gerontology. Series B, Psychological Sciences and Social Sciences, 74(5), 796-805. doi: https://doi.org/10.1093/geronb/gbx137.

Publisher's Note Springer Nature remains neutral with regard to jurisdictional claims in published maps and institutional affiliations. 